\title{
ON CERTAIN CONSTRUCTIONS IN FINITISTIC SPACES
}

\section{SATYA DEO and MOHAN SINGH}

\author{
Department of Mathematics \\ University of Jammu \\ Jammu - 180001, INDIA
}

(Received April 23, 1982 and in revised form November 25, 1982)

ABSTRACT. Since the product of two finitistic spaces need not be finitistic, and also because a continuous closed image of a finitistic space need not be finitistic, it is natural to enquire whether or not the class of finitistic spaces in closed under the formation of cones, reduced cones, suspensions, reduced suspensions, adjunction spaces, mapping cylinders, mapping cones, joins and smash products. In this paper we prove that all of the above constructs, except joins and smash products, of finitistic spaces are finitistic. The joins and smash products of finitistic spaces, however, need not be finitistic. We find sufficient conditions under which these are also finitistic.

KEY WORDS AND PHRASES. Finitistic spaces, adjunction spaces, covering dimension. 1980 MATHEMATICS SUBJECT CLASSIFICATION CODE. Primary 54B17, Secondary 54 F45.

1. INTRODUCTION AND PRELIMINARIES.

A paracompact Hausdorff space $X$ is said to be finitistic (or satisfies Swan's condition [1]) if each open cover has a finite-dimensional open refinement. These spaces have proved to be quite useful in the cohomology theory of topological transformation groups (Cf. [2], [3], [4]). Analogous to the classical P.A. Smith theorems, there are quite nice and interesting results about group actions on those finitistic spaces which are cohomology (or homotopy) spheres, projective spaces, Poincare duality spaces, etc. The basic techniques of homology-cohomology and homotopy theories for finitistic spaces are, therefore, natural prerequisites. The first few topological results, one would like to know in this direction are whether or not certain constructs like cones, reduced cones, suspensions, reduced suspensions, adjunction spaces, mapping cylinders, mapping cones, joins and smash products involving finitistic spaces are again finitistic spaces.

It was pointed out in [5] that product of even two finitistic spaces need not be finitistic. In this paper we give an example to show that product of even a compact space and a finite-dimensional space need not be finitistic. This example, incidently, 
leads us to find out some necessary and some sufficient conditions for the product of two finitistic spaces to be finitistic. One interesting result in this direction is the theorem 2.2, which states that the product of a finitistic space and a compact finite-dimensional space is finitistic. Conversely, if X X Y is finitistic for each finitistic space $Y$ then $X$ must be compact and finite-dimensional. It turns out that the converse follows from our example and a theorem of Arens-Dugundji ([6], p. 229). The direct part, in particular, tells us that the cylinder over a finitistic space is finitistic. Since a continuous closed image of a finitistic space need not be finitistic [Cf. 5], we cannot conclude that cones, reduced cones, suspensions, reduced suspensions, mapping cylinders, mapping cones, and adjunction slaces constructed from finitistic spaces are finitistic. But, then the following theorem which has been proved in [5] can be used to show that, indeed, we can conclude that all of the above constructs of finitistic spaces are finitistic.

THEOREM 1.1. A paracompact Hausdorff space is not finitistic if and only if there exists a discrete family $\left\{A_{n}\right\}_{n=0}^{\infty}$ of closed sets such that $\operatorname{dim} A_{n}>n$, for each $n$.

The fact that the adjunction space of finitistic spaces is finitistic combined with the results about products of finitistic spaces yields some necessary as well as some sufficient conditions for the join of two finitistic spaces to be finitistic, and the same conditions turn out to be sufficient for the smash product of finitistic spaces to be finitistic.

Let $\mathrm{X}$ be any topological space and $\mathrm{I}$ denote the closed unit interval. Then by identifying the top $\mathrm{X} \times\{1\}$ of the product $\mathrm{X} \times \mathrm{I}$ to a single point, we get the cone $C(X)$ over the space $X$. In addition, if we identify the bottom $X x\{c\}$ also to a single point we get the suspension $S(X)$ of the space $X$. If we take pointed space $\left(X, x_{0}\right)$ and identify $(X \times\{1\}) \cup(\{x\} \quad x$ I) to a single point, we get so called reduced cone, and similarly if $\left.\left(X \times\left\{{ }_{1}\right\}\right) \cup\left(\left\{x_{0}\right\} \times x\right) \cup\left(X \times{ }_{0}\right\}\right)$ is identified to a point, we get the reduced suspension $\mathrm{S}(\mathrm{X})$. As is well known all these constructions are special cases of the general concept of adjunction space. If $X, Y$ are two spaces, $A \subset X$ is closed and $f$ : $A \rightarrow Y$ is continuous, then the quotient space obtained by identifying a $\varepsilon$ A with $f(a)$ in the disjoint union $X / J Y$ is called the adjunction space and is denoted by $X \cup_{f} Y$. The mappsig cylinder $\mathrm{Z}(\mathrm{f})$ of a given map $\dot{\mathrm{f}}: \mathrm{X} \rightarrow \mathrm{Y}$ is the adjunction space of $\mathrm{X} X \mathrm{X}$ to $\mathrm{Y}$ by $f: X x i o\}, Y$, and the mapping cone $C(f)$ is the adjunction space of $C(X)$ to $Y$ by $\mathrm{f}: \mathrm{X}$ xiof $\rightarrow \mathrm{Y}$. we also recall that if $\left(\mathrm{X}, \mathrm{x}_{\mathrm{O}}\right),\left(\mathrm{Y}, \mathrm{y}_{\mathrm{O}}\right)$ are two pointed spaces and if in the prow..e $\therefore \times x$ we identify $\left(X \times\left\{y_{0}\right\}\right) \cup\left(\left\{x_{0}\right\} \times x\right)$ to a single point, the quotient

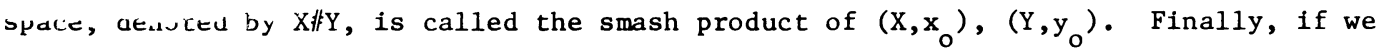
consider cue product X X I X Y and make the following identifications, then the quucienc space so obtained is called the join of $\mathrm{X}$ and $\mathrm{Y}$ and is denoted by $\mathrm{X} * \mathrm{Y}$ : $(\mathrm{x}, u, j, \cdots \mathrm{x}$ and $(\mathrm{x}, 1, \mathrm{y}) \sim \mathrm{y}$, where $\mathrm{x} \varepsilon \mathrm{X}, \mathrm{y} ; \varepsilon \mathrm{Y}$.

.. *UUUCT OF FINITISTIC SPACES

kecall that a paracompact Hausdorff space $X$ is said to be finitistic if each open 
cover of $\mathrm{X}$ has a finite-dimensional open refinement, (Cf.[5] for details). Let us consider the following:

EXAMPLE 2.1. Suppose $I^{\omega}$ stands for the infinite-dimensional Hilbert-cube, and let $\cup_{i \geq 1} I_{i}^{\omega}$ be the disjoint union of denumerable copies of such Hilbert-cubes. Suppose $\mathrm{N}=\{1,2,3, \ldots\}$ is the space of natural numbers with discrete topology. Then $N$ is a zero-dimensional metric space and $I^{\omega}$ is compact. However, the product $I^{\omega} x N$, being homeomorphic to $\cup_{i \geq 1} I_{i}^{\omega}$ is not finitistic by theorem 1.1 .

The following weaker form of this example will be frequently used in the sequel: If $X$ is any infinite dimensional space and $N$ is the discrete space of natural numbers, then $\mathrm{X} \times \mathrm{N}$ is never finitistic.

Recall that each compact space and each paracompact space of finite covering dimension is finitistic. The above example shows not only that the product of two finitistic spaces need not be finitistic but much more, viz., that the product of even a compact space with a finite-dimensional space need not be finitistic. In the above example $I^{\omega}$ is compact and infinite-dimensional. One cannot have a stronger example because of the following:

THEOREM 2.2. Let $X$ be a compact finite-dimensional space. Then X $X Y$ is finitistic for each finitistic space $Y$. Conversely, if X X Y is finitistic for each finitistic space $Y$ then $X$ must be compact and finite-dimensional.

PROOF. Let $u$ be an open cover of $X \times x$. For $(x, y) \varepsilon X \times Y$, there exist $U \varepsilon u$, $\mathrm{U}_{\mathrm{x}}$ open in $\mathrm{X}$ and $\mathrm{V}_{\mathrm{y}}$ open in $\mathrm{Y}$ such that $(\mathrm{x}, \mathrm{y}) \varepsilon \mathrm{U}_{\mathrm{x}} \mathrm{x} \mathrm{V}_{\mathrm{y}} \sim \mathrm{U}$. For each $\mathrm{y} \varepsilon \mathrm{Y},\left\{\mathrm{U}_{\mathrm{x}} \mid \mathrm{x} \varepsilon \mathrm{X}\right\}$ is an open cover of $x$. Since $x$ is compact, there exists $\left\{U_{y_{j}} \mid j=1,2, \ldots, n_{y}\right\}$ a finite subcover of $\left\{U_{x} \mid x \in X\right\}$. Let $v_{y}=\bigcap_{j=1}^{n} v_{y_{j}}$. Then $\left\{U_{y_{j}} x v_{y} \mid j=1,2, \ldots, n y, y \varepsilon Y\right\}$ is an open refinement of $u$. Let $\operatorname{dim} X \leq n$. For each y $\varepsilon Y,\left\{U_{y_{j}} \mid j=1,2, \ldots, n_{y}\right\}$ has an open refinement $\left\{\mathrm{H}_{y_{j}} \mid j=1,2, \ldots, n_{y}\right\}$ of dimension not exceeding $n$. Since $Y$ is finitistic, there exists an open refinement $\left\{G_{y} \mid y \varepsilon Y\right\}$ of $\left\{V_{y} \mid y \varepsilon Y\right\}$ of finitedimension, say m. Then

$$
\left\{H_{y_{j}} \times G_{y} \mid j=1,2, \ldots, n_{y}, y \in Y\right\}
$$

is an open refinement of $u$ of dimension not exceeding $\mathrm{mn}$.

Conversely, first we show that $X$ is compact. Since X X Y is finitistic, X being a closed subset of $\mathrm{X} \times \mathrm{Y}$, must be finitistic. So in particular $\mathrm{X}$ is paracompact. Now to show that $X$ is compact it suffices, by a Theorem of Arens-Dugundji ([6],p.229) to show that $X$ is countably compact. Suppose $X$ is not countably compact. Then by Bolzano-Weierstrass property there exists a countably infinite set $A=\left\{a_{1}, a_{2}, \ldots, a_{n}\right.$, ...\} which has no limit point, i.e., A is an infinite closed, discrete set. Now if we take $Y=I^{\omega}$, then $A X Y$ is not finitistic. Thus we have a contradiction. Also X must be finite-dimensional. For, otherwise on taking $\mathrm{Y}=\{0,1, \ldots, \mathrm{n}, \ldots\}$ we $\mathrm{find}$ that 
$\mathrm{X} \times \mathrm{Y}$ is not finitistic.

When we consider the product $X \times Y$ of two finitistic spaces $X$ and $Y$, the first difficulty in having $X \times \mathrm{Y}$ finitistic is that it need not be paracompact because product of even two paracompact spaces need not be paracompact. Thus the problem of analyzing when the product will be finitistic will be examined after making the assumption that the product is already paracompact. The following result 1 ists all possibilities when $\mathrm{X} \times \mathrm{Y}$ is assumed to be finitistic.

PROPOSITION 2.3. Let $X, Y$ be two non-empty spaces. If the product $X X Y$ is finitistic then one of the following must hold:

(a) One is compact finite-dimensional and the other is finitistic,

(b) One is compact infinite-dimensional and the other is compact,

(c) One is non compact finite-dimensional and the other is finite-dimensional. Conversely, each of (a) and (b) implies that X X Y is finitistic.

PROOF. Suppose $\mathrm{X} \times \mathrm{Y}$ is finitistic. Then $\mathrm{X}$ and $\mathrm{Y}$ being homeomorphic to closed subspaces of $\mathrm{X} \times \mathrm{Y}$ are also finitistic. For any space $\mathrm{X}$ one and only one of the following four conditions must be satisfied:

(i) $\mathrm{X}$ is compact and finite-dimensiona1,

(ii) $\mathrm{X}$ is compact and infinite-dimensional,

(iii) $X$ is non-compact and finite-dimensional,

(iv) $\mathrm{X}$ is non-compact and infinite-dimensional.

Now, if $\mathrm{X}$ is compact and finite-dimensional, then obviously (a) holds and there is nothing to prove. If $\mathrm{X}$ is compact infinite-dimensional, then we claim that $\mathrm{Y}$ must be compact. For, otherwise one can find a discrete closed subset $B$ of $Y$ which is homeomorphic to $\mathrm{N}$. This will mean that $\mathrm{X} \times \mathrm{B}$ is closed in $\mathrm{X} \times \mathrm{Y}$. But then, just as in example 2.1, X X B is not finitistic, and X $\mathrm{X} Y$ cannot be finitistic - a contradiction. Hence (b) holds. If $X$ is non-compact finite-dimensional, then we claim that $Y$ must be finite-dimensional. Since $X$ is non-compact, there is a closed discrete subset $A$ of $X$ homeomorphic to $\mathrm{N}$. Hence if $\mathrm{Y}$ is infinite-dimensiona1, A $x \mathrm{Y}$ will be non-finitistic closed subspace of $\mathrm{X} \times \mathrm{Y}$, and therefore $\mathrm{X} \times \mathrm{Y}$ cannot be finitistic - again a contradiction. Hence we are in (c). Finally if $X$ is non-compact infinite-dimensional, then by similar arguments one can easily prove that $Y$ must be compact as well as finitedimensional, and hence we are back in (a).

Conversely, (a) implies that $\mathrm{X} \times \mathrm{Y}$ is finitistic, follows from theorem 2.2 and (b) implies that $\mathrm{X} \times \mathrm{Y}$ is compact, which means $\mathrm{X} \times \mathrm{Y}$ is finitistic.

REMARK 2.4. Whether or not (c) in theorem 2.3 is a sufficient condition for $X \times Y$ to be finitistic, appears to be a hard problem about the product of finitistic spaces. Precisely, the problem is the following: Is it true that the product of two non-compact finite-dimensional paracompact spaces is finitistic? If the paracompact product of two paracompact finite-dimensional spaces were finite-dimensional, then the above problem was trivial since each finite-dimensional paracompact space is finitistic. But in this generality, that again is an open problem. One may, however, say that even if the 
paracompact product of two finite-dimensional spaces is not finite-dimensional, yet it may be finitistic. The following result shows that this cannot happen.

PROPOSITION 2.5. Either the paracompact product of two finite-dimensional paracompact spaces is finite-dimensional or else there exist two paracompact finite-dimensional spaces whose product is paracompact but not finitistic.

PROOF. Suppose there exist two paracompact finite-dimensional spaces $X$ and $Y$ such that $\mathrm{X} \times \mathrm{Y}$ is paracompact infinite-dimensional. Let $\mathrm{Z}=\mathrm{X} \times\{0,1,2, \ldots\}$. Now $\mathrm{Z}$ and $\mathrm{Y}$ are two finite-dimensional paracompact spaces such that $\mathrm{Z} \times \mathrm{Y}$ is paracompact but not finitistic. By a similar argument we can prove that if for all paracompact finite-dimensional spaces $X$ and $Y, X \times Y$ is finitistic, then $X \times Y$ is finite-dimensional.

\section{ADJUNCTION SPACES OF FINITISTIC SPACES}

Recall that if $\mathrm{X}, \mathrm{Y}$ are two spaces, $\mathrm{A} \subset \mathrm{X}$ is closed and $\mathrm{f}: \mathrm{A} \rightarrow \mathrm{Y}$ is a continuous map then the space $X L_{f} \dddot{i}$ obtained by identifying a $\varepsilon$ A with $f(a)$ in the disjoint union $X \cup Y$ is called the adjunction space of $X$ to $Y$ by the map $f$. First of all we have

THEOREM 3.1. Suppose $X, Y$ are finitistic spaces, $A \subset X$ is a closed set and $f: A \rightarrow Y$ is a continuous map. Then the adjunction space $\mathrm{X}()_{\mathrm{f}} \mathrm{Y}$ is also finitistic.

PROOF. Because X,Y are paracompact Hausdorff spaces, the adjunction space $X \cup_{f} Y$ must be paracompact Hausdorff ([6],Ex.3,p.178). Recall that a closed subset of a finitistic space is again finitistic. Note that $Y$ is embeded as a closed subset of $\mathrm{X} \cup_{\mathrm{f}} \mathrm{Y}$, and any closed subset of $\mathrm{X} \cup_{\mathrm{f}} \mathrm{Y}$ which is disjoint from $\mathrm{Y}$ is finitistic because it is, then, a closed subset of $X$. Hence the proof of the theorem follows at opce from the following:

PROPOSITION 3.2. Suppose $X$ is a paracompact Hausdorff space and $F$ is a closed subset of $X$. If $F$ is finitistic and every closed subset of $X$ which is disjoint from $F$ is also finitistic, then $X$ itself is finitistic.

PROOF. Suppose $X$ is not finitistic. Then by theorem 1.1 there exists a discrete family $\left\{A_{n}\right\}_{n=0}^{\infty}$ of closed subsets of $X$ such that for each $n, \operatorname{dim} A_{n}>n$. Since the family $\left\{A_{n} \cap F\right\}_{n=0}^{\infty}$ is again a discrete family of closed sets of $F$ and since $F$ is finitistic, the set $\left\{\operatorname{dim}\left(A_{n} \cap F\right)\right\}_{n=0}^{\infty}$ must be bounded, say by $m$. Let $n>m$. Since $\operatorname{dim} A_{n}>n, \operatorname{dim}\left(F \cap A_{n}\right)$ $\leq \mathrm{m}<\mathrm{n}$ and $\mathrm{F} \cap \mathrm{A}_{\mathrm{n}}$ is closed in $\mathrm{A}_{\mathrm{n}}$, it follows from ([7],p.136) that there exists a set $B_{n}$ which is disjoint from $A_{n} \cap F$ and $\operatorname{dim} B_{n}>n$. Let $C_{n}=B_{m+n+1}$. The family $\left\{C_{n}\right\}_{n=0}^{\infty}$ is a discrete family of closed subsets of $X$ such that $\operatorname{dim} C_{n}>n$. This means that the set $E=\bigcup_{n=0}^{\infty} C_{n}$ is a closed set disjoint from $F$ which is not finitistic. This is a contradiction.

COROLLARY 3.3. The class of finitistic spaces is closed under the formation of cones, reduced cones, suspensions, reduced suspensions, mapping cylinders, mapping cones, and adjunction spaces. 
COROLLARY 3.4. Let $X, Y$ be two non-empty spaces. If the join $X * Y$ is finitistic then one of the following must hold:

(a) One is compact finite-dimensional and the other is finitistic,

(b) One is compact infinite-dimensional and the other is compact,

(c) Both are non-compact finite-dimensional.

Conversely, each of (a) and (b) implies that the join X * Y is finitistic. Further-

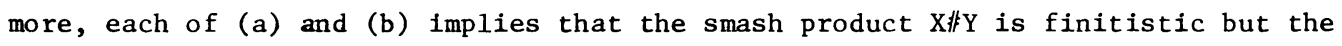
converse is not true.

\section{REFERENCES}

1. SWAN, R.G. A New Method in Fixed Point Theory, Comm. Math. Helv. 34 (1960), 1-16.

2. BREDON, G.E. Introduction to Compact Transformation Groups, Academic Press (1972).

3. DEO, S. and SINGH, T.B. On the Converse of Some Theorems About Orbit Spaces, J. London Math. Soc. (2) 25 (1982), 162-170.

4. HSIANG, W.Y. Cohomology Theory of Topological Transformation Groups, SpringerVerlag, Ergebnisee, (1975).

5. DEO, S. and TRIPATHI, H.S. Compact Lie-Group Actions on Finitistic Spaces, Topology 21 (1982), 393-399.

6. DUGUNDJI, J. Topology, A11yn and Bacon, Inc., (1960).

7. PEARS, A.R. Dimension Theory of General Spaces, Cambridge Univ. Press (1975). 


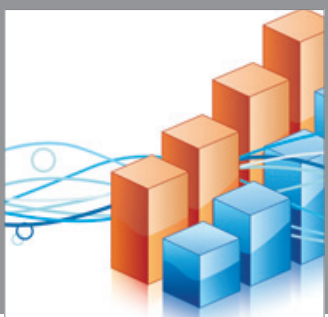

Advances in

Operations Research

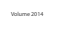

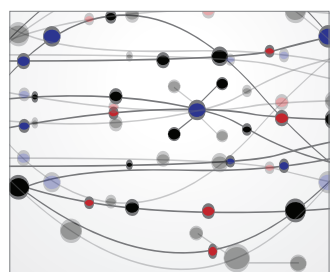

\section{The Scientific} World Journal
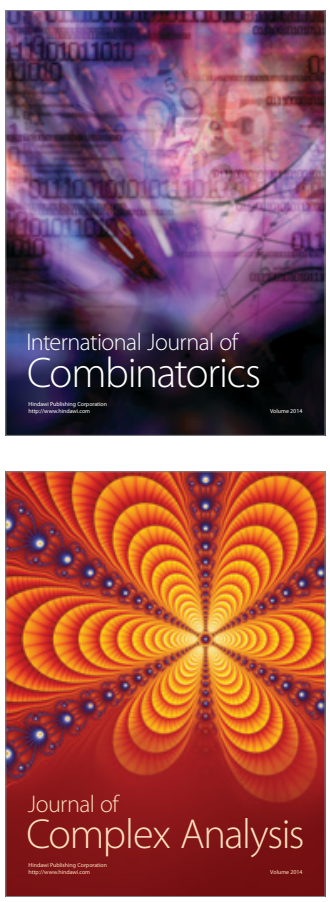

International Journal of

Mathematics and

Mathematical

Sciences
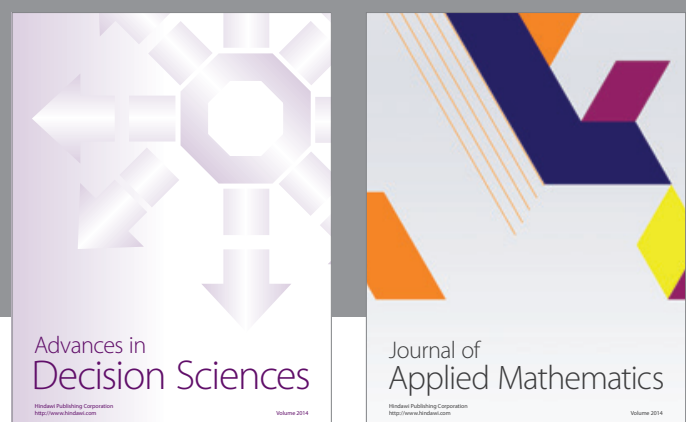

Journal of

Applied Mathematics
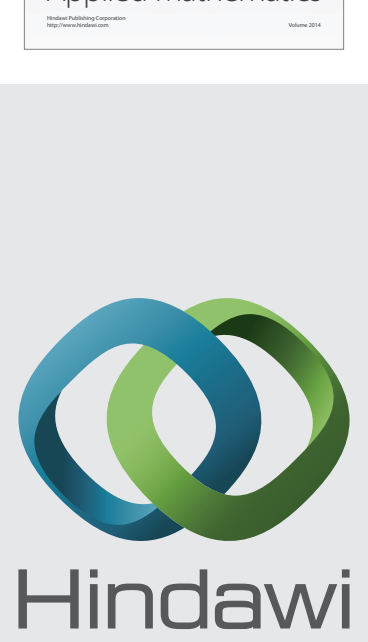

Submit your manuscripts at http://www.hindawi.com
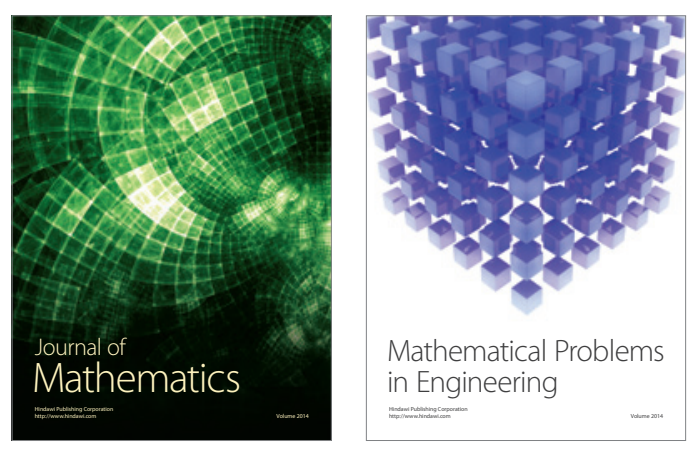

Mathematical Problems in Engineering
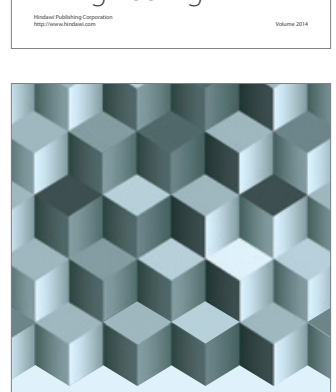

Journal of

Function Spaces
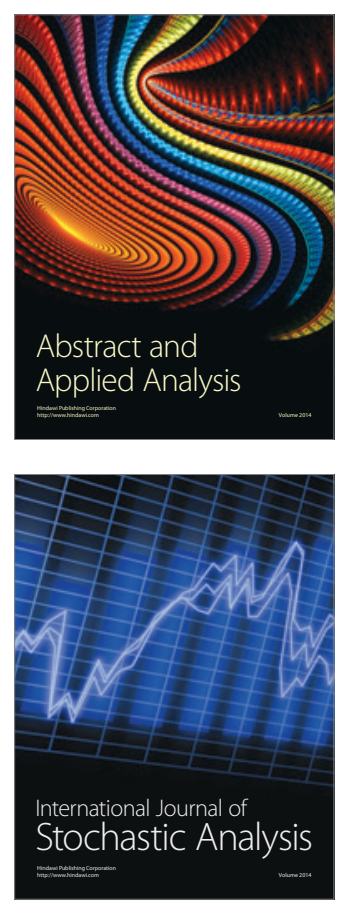

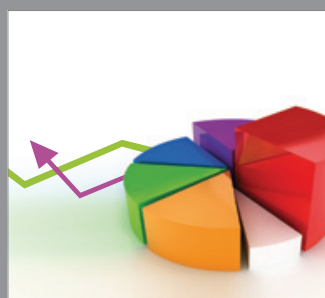

ournal of

Probability and Statistics

Promensencen
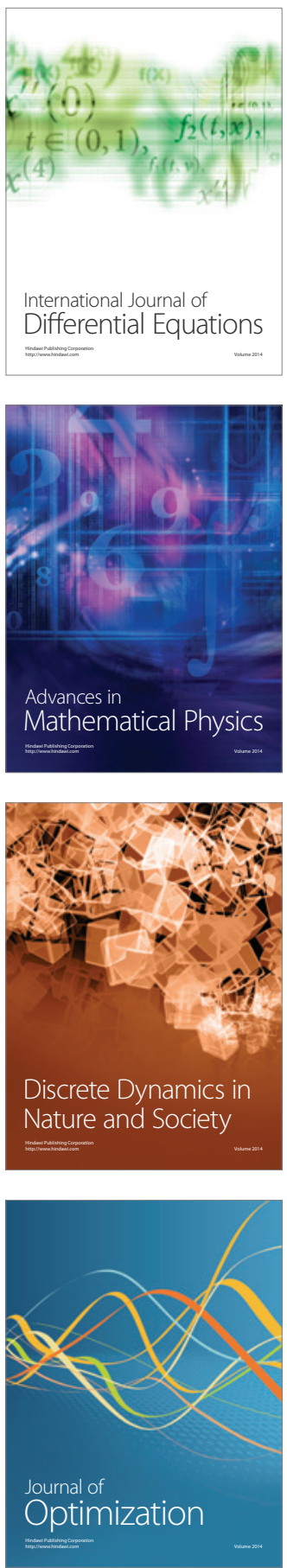